\title{
CERVICAL CANCER PREVENTION IN SETTINGS OF HIGH HIV PREVALENCE
}

\author{
Sonia Menon, $M P H, M A, P G D$ in Infectious Diseases \\ London School of Hygiene and Tropical Medicine Alumni, London, UK
}

Despite being a preventable disease, cervical cancer is still the second most common cancer in women worldwide. HIV infection is associated with a higher incidence, more rapid progression, and increased recurrence rates of human papillomavirus (HPV)-associated cervical intra-epithelial neoplasia and invasive cancer. The disease burden in developing countries is the result of inadequate national health care infrastructures that cannot establish or sustain comprehensive screening programmes, together with a high prevalence of HIV infection, particularly in southern Africa. In this article, clinically relevant issues for primary prevention of cervical lesions by a quadrivalent HPV vaccine and the 'screen-and-treat' protocol in settings of high HIV prevalence will be explored.

\section{HIV AND HPV CO-INFECTION}

HIV is believed to increase the risk of HPV infection and cervical neoplasia, in part due to HIV-induced immunodeficiency and the resulting inability to control HPV infection. ${ }^{1,2}$ HIV status, herpes zoster, oral candidiasis and tuberculosis have all been found to be associated with carcinogenic human papillomavirus (HPV), consistent with other studies that found HIV and HIV-associated immunosuppression to be independently associated with HPV positivity. ${ }^{3}$

Although it has been recognised that HIV plays a significant role in increasing the risk of persistent/latent HPV infection and/or rates of progression of precancerous lesions to high-grade cervical neoplasia and cancer, several key variables remain to be elucidated. These include a better understanding of the role of HPV viral load in the genesis of cervical neoplasia in HIV-infected women, and the relationship between early initiation of HAART and the possible inadvertent result of an increased risk of the acquisition of molecular changes characteristic of carcinoma in situ.

In contrast to a very small subset of the many immunocompetent women infected with oncogenic types of HPV who develop cervical cancer, women infected with HIV are thought to be 3 - 5 times more likely to develop cervical lesions that can become cancerous. ${ }^{4}$ Both pre-invasive disease and invasive cervical cancer have been reported to have a much poorer outcome in HIV-infected women than in the general population. ${ }^{5}$

Similar to cervical disease progression, recurrent disease after treatment is correlated with low CD4 cell counts. ${ }^{6}$ Although at least seven studies have examined the effects of highly active antiretroviral therapy (HAART) on the course of cervical lesions, it is still not clear whether HAART substantially affects the natural history of cervical squamous intra-epithelial lesions (SIL). The impact of HAART has led to some improved resolution of abnormal Pap smears, but has not made a significant impact on the risk of cervical cancer in HIV-infected women. ${ }^{7}$ This may be because administration of HAART is most often offered to women with more advanced HIV disease status and higher HPV viral loads. HAART also prolongs survival in women with cervical pre-invasive lesions. $^{8}$

In this article, it will be argued that while a 'one-visitin-a-lifetime' strategy with immediate cryotherapy or the loop electrosurgical excision procedure (LEEP) as part of the minimum package for the high-risk age group of women between 35 and 50 years may have an impact in resource-poor settings, this protocol in areas of high HIV prevalence may not take into account the high rates of recurrence and cervical disease progression associated with low CD4 cell counts. Secondly, it will be explored how a HPV DNA-based screening programme in high HIV prevalence areas may result in overtreatment because of its low specificity. Finally, it will be argued why a vaccinebased cervical cancer prevention programme may not be sufficient to reduce cervical cancer in southern Africa.

\section{DISCUSSION}

\section{SCREEN-AND-TREAT PROTOCOL IN SETTINGS WITH HIGH} PREVALENCES OF HIV AND GENITAL ULCERATION

In developing countries, inadequate screening programmes have contributed to high incidences of cervical cancer. While in developed countries the introduction of large-scale cytological testing has resulted in a major decline in cervical cancer mortality, in low-resource settings the high specificity of cytological testing is offset by its lack of sensitivity for detection of precursors of invasive cervical cancer (ranging from 30\% to $90 \%$ ) and highly dependent on adequacy of sample collection, slide preparation and slide interpretation.

As part of an efficient cervical prevention programme, alternatives to cervical cytology have been sought but have not met a high level of specificity. Several recent 
studies have demonstrated that direct visual inspection of the cervix with acetic acid (VIA) is a reasonably sensitive and a cost-effective alternative to cytological screening. ${ }^{9}$ With a sensitivity of $76 \%$ in HIV-positive women, VIA is also a useful screening test for pre-invasive lesions of the cervix in low-resource settings. ${ }^{10}$ However, the high prevalence of sexually transmitted infection (STI)-related genital ulcers in the African countries worst affected with HIV may lead to a relatively low specificity of VIA. ${ }^{11}$

HPV DNA testing has emerged as a convincing option for cervical cancer screening. A large study in India in 1999 on healthy women aged between 30 and 59 years found that a programme strategy based on a single round of HPV testing was associated with a 50\% reduction in cervical cancer incidence and mortality, whereas strategies based on a single round of VIA or Pap screening had little, if any, effect on these outcomes. ${ }^{12}$ It was recommended that since most HPV infections in young women regress rapidly without causing clinically significant disease, a single HPV testing round would be associated with a significant reduction in the numbers of advanced cervical cancers and death from cervical cancer if targeted at women aged over 30 years. ${ }^{12}$

A randomised controlled trial in South Africa in 2000 2002 led to recommendations that both VIA and HPVbased screening and immediate cryotherapy treatment were safe and decreased the prevalence of high-grade cervical cancer precursor lesions. ${ }^{13}$ Several studies have been undertaken in low-resource settings to assess the optimal age group for cervical cancer screening to achieve the greatest public health impact. A costeffectiveness modelling exercise comparing screening strategies in five developing countries predicted that for 35-year-old women screened only once in their lives, a 1 2-visit approach with the VIA method could reduce the lifetime risk of cervical cancer by $25 \%$, and HPV DNA testing could reduce it by $36 \% .{ }^{14}$

Although these recommendations may be effective in HIV-negative women, in whom there is the possibility of spontaneous regression of pre-invasive lesions because of their normally functioning immune systems, certain issues would need to be explored in resource-poor settings with a high prevalence of HIV.

HIV-positive women have high rates of SIL and concurrent HPV infections with a variety of genotypes in which the oncogenic risk is poorly documented. A high diversity of HPV genotypes was observed in HIV-infected women in Brazil. Many of these women infected with HPV were found to carry oncogenic genotypes, even when cytological evaluation showed normal results. ${ }^{15}$

Although HPV testing of cervical smears is more sensitive than cytological assessment, the specificity of HPV DNA testing may be unacceptably low in areas of high HIV prevalence. In one study, the specificity of HPV DNA testing for detection of high-grade squamous intraepithelial lesions (HSIL) was $75 \%$ in HIV-seronegative women and $41 \%$ in HIV-seropositive women. ${ }^{16}$
In HIV-positive women, rapid progression of HPV may reduce the age at which women are 'at high risk'. In a study to determine the effect of the HIV epidemic on invasive cervical cancer in Kenya, HIV-positive women who presented with cervical cancer were found to be significantly younger than HIV-negative women. ${ }^{17}$ These findings imply that screening of 35-year-old women only once in their lives may not reduce the lifetime risk of cervical cancer in a high HIV prevalence setting.

In Zambia, where the prevalence of HIV infection is one of the highest in the world and the incidence of cervical cancer the highest in sub-Saharan Africa, ${ }^{18}$ it was found that in a resource-constrained environment it would be feasible to implement a system of referral of cryotherapy-ineligible patients for LEEP in a 'screenand-treat' cervical cancer prevention programme targeting HIV-infected women. ${ }^{19} \mathrm{~A}$ study conducted in Thailand between 2004 and 2008 demonstrated that HIV infection was not significantly associated with LEEP complications. ${ }^{20}$

\section{PROPHYLAXIS-BASED SECONDARY CERVICAL CANCER PREVENTION PROGRAMME}

Settings of high HIV prevalence such as Rakia, Uganda, where almost $50 \%$ of HIV-positive women are infected with strains of HPV that are associated with a risk of cervical cancer, ${ }^{21}$ would stand to benefit most from a prophylaxis-based (vaccine) cervical cancer prevention programme aimed at HPV-naïve women.

A robust surveillance system capable of monitoring long-term safety, sustained immune responses, vaccine efficacy, and the epidemiological distribution of HPV oncogenic strains also encountered in HIV-positive women would be needed. Although the quadrivalent HPV vaccine against HPV $6,11,16$ and 18 constitutes an important breakthrough in cervical cancer control in HIV-negative women in the developed world, the limited epidemiological data available suggest that a much wider variety of HPV types are involved in the pathogenesis of cervical neoplasia in developing countries. ${ }^{22}$ Also, HIVinfected women in various geographical regions, such as Zambia, Brazil and Rochester, NY, appear to be infected with less prevalent types of HPV compared with the general population. ${ }^{23}$

Evidence suggests that different HPV types behave as independent infections, with no cross-reactive cellmediated immunity that might potentially be able to keep the oncogenic non-vaccine types under control. In 2009 a double-blind randomised study in young women indicated that the immune response stimulated by HPV 16 and 18 may also confer individual cross-protection against genetically related HPV oncogenic types, such as HPV 31,33 and $45 .{ }^{24}$ Monitoring would be necessary to see whether individual cross-protection could extend some protection to HIV-infected women in sub-Saharan Africa, and whether the Af variants of HPV 16 and 18 common in women of African descent ${ }^{25}$ impact on the effectiveness of the HPV 16 and 18 vaccine and on individual cross-protection. 


\section{CONCLUSION}

This paper has explored issues regarding screening for cervical cancer in settings with a high prevalence of HIV and genital ulceration. While the new HPV test has proved to be a successful cancer screening tool in detecting 14 high-risk types of carcinogenic HPV with $90 \%$ accuracy when tested on a group of local women in Eastern China, ${ }^{26}$ its effectiveness will also need to be assessed in a high HIV prevalence setting to ascertain its specificity and sensitivity in HIV-positive women.

Although in Zambia piggybacking on established HIV infrastructure has optimised resources and increased the efficiency of both HIV and cervical cancer programmes, ${ }^{27}$ the once-in-a-lifetime screen-and-treat protocol focusing on women between 30 and 45 years of age recommended for sub-Saharan Africa may not adequately take into account what is already known about the epidemiology of HIV-HPV co-infection. Longer follow-up will be needed to assess the efficacy, cost-effectiveness and safety of VIA with same-visit treatment with LEEP in HIV-positive women.

It is important to keep in mind that the prophylaxis prevention programme would be implemented in a setting where malnutrition and decreased immunity due to HIVIAIDS result in far less spontaneous regression of cervical lesions than would be seen in industrialised countries. This fact underscores the need for prevailing strains to be fully characterised and linkage to be established between vaccination history, screening history and HPV exposure.

\section{REFERENCES}

1. Robinson WR, Freeman D. Improved outcome of cervical neoplasia in HIV-infected women in the era of highly active antiretroviral therapy. AIDS Patient Care STDs 2002;16(2):61-65. http://www.ncbi.nlm.nih.gov/pubmed/11874637 (accessed 14 December 2009).

2. McKenzie ND, Kobetz EN. Women with HIV are more commonly infected with non-16 and -18 high-risk HPV types. Gynecol Oncol 2010;116:572-577.

3. Carter M. High prevalence of cervical cancer associated HPV strains in HIV-positive women. 2008. http://www.womensnet.org.za/high-prevalence-cervical-cancer-associatedhpv-strains-hivpositive-women (accessed 10 March 2010).

4. PlusNews. Global HIVIAIDS news and analysis. South Africa: Cervical cancer vaccine offers distant hope. 19 June 2007. http://www.plusnews.org/report.aspx?ReportID=72809 (accessed 10 March 2010)

5. Robinson WR, Freeman D. Improved outcome of cervical neoplasia in HIV-infected women in the era of highly active antiretroviral therapy. AIDS Patient Care STDs 2002;16(2):61-65. http://www.ncbi.nlm.nih.gov/pubmed/11874637 (accessed 14 December 2009).

6. Sweet RL, Gibbs RS. Infectious Diseases of the Female Genital Tract. 5th ed. Philadelphia: Lippincott Williams \& Willkins, 2009.

7. Cameron JE, Hagensee ME. Human papillomavirus infection and disease in the HIV+ individual. Cancer Treat Res 2007;133:185-213. http://www.springerlink.com/content/ g12t34x256681832/ (accessed 10 February 2010).
8. Xi LF, Kiviat NB. Cervical neoplasia and highly active antiretroviral therapy. J Natl Cancer Inst 2004;96(14):1051-1053. http://jnci.oxfordjournals.org/cgi/reprint/96/14/1051 (accessed 7 January 2010).

9. Goldie SJ, Kuhn L, Denny L, Pollack A, Wright TC. Policy analysis of cervical cancer screening strategies in low-resource settings. JAMA 2001;285:3107-3115. http://jama.amaassn.org/cgi/content/full/285/24/3107 (accessed 10 November 2009).

10. Denny L, Kuhn L, Pollack A, Wainwright H, Wright TC Jr. Evaluation of alternative methods of cervical cancer screening for resource-poor settings. Cancer 2000:89:826-833. http:/l www.ncbi.nlm.nih.gov/sites/entrez?cmd=search\&db=pubmed\&term=10951346 (accessed 20 November 2009).

11. Walraven G. Prevention of cervical cancer in resource-poor settings. Lancet 2009;361:21602161

12. Sankaranarayanan R, Nene BM, Shastri SS, et al. HPV screening for cervical cancer in rural India. N Engl J Med 2009;360:1385-1394. http://content.nejm.org/cgi/content/full/360/ 14/1385?ijkey=468a8b491975664292c6caae48a7a3e86b057962 (accessed 24 November 2009).

13. Kuhn L, Wang C, Tsai W-Y, Wright TC, Denny L. Efficacy of human papillomavirusbased screen-and-treat for cervical cancer prevention among HIV-infected women. AIDS 2010;24:2553-2561. http://www.ph.ucla.edu/epi/faculty/detels/Epi293/CervCancer_ AIDS_2010.pdf (accessed 1 January 2010).

14. Goldie SJ, Gaffikin L, Goldhaber-Fiebert JD, et al Cost-effectiveness of cervical-cancer screening in five developing countries. N Engl J Med 2005;353(20):2158-2168. http:/l content.nejm.org/cgi/content/full/353/20/2158 (accessed 29 November 2009).

15. Cerqueira DM, Moraes DS, Camara GN, et al. High HPV genetic diversity in women infected with HIV-1 in Brazil. Arch Virol 2007;152:75-83. http://www.springerlink.com/content/ h47682624327j607/ (accessed 10 March 2010).

16. Belinson J, Qiao Y, Pretorius R, et al. Prevalence of cervical cancer and feasibility of screening in rural China: a pilot study for the Shanxi Province Cervical Cancer Screening Study. Int J Gynecol Cancer 1999;9:411-417.

17. Gichangi P, De Vuyst H, Estambale B, et al. HIV and cervical cancer in Kenya. Int J Gynecol Obstet 2002:76(1):55-63.

18. PlusNews. Global HIVIAIDS news and analysis: Zambia (2009). Treating cervical cancer and HIV simultaneously. http://www.plusnews.org/Report.aspx?Reportld=85961 (accessed 27 April 2011).

19. Pfaendler KS, Mwanahamuntu MH, Sahasrabuddhe VV, Mudenda V, Stringer JSA, Parham GB. Management of cryotherapy-ineligible women in a 'screen-and-treat' cervical cancer prevention program targeting HIV-infected women in Zambia: Lessons from the field. Gynecol Oncol 2008;110:402-407. http://web.cs.swarthmore.edu/ turnbull/cs91/f09/paper/ pfaendler08.pdf (accessed 31 Jaunary 2010)

20. Kietpeerakool C, Suprasert P, Srisomboon J. Outcome of loop electrosurgical excision for HIV-positive women in a low-resource outpatient setting. Int J Gynecol Obstet 2009;105(1):10-13.

21. Safaeian M, Kiddugavu M, Gravitt PE, et al. Prevalence and risk factors for carcinogenic human papillomavirus infection in rural Rakai, Uganda. Sex Transm Infect 2008;84:306-311. http://sti.bmi.com/content/84/4/306 (accessed 14 January 2010).

22. Bosch FX, Manos MM, Munoz N, et al. Prevalence of human papillomavirus in cervical cancer: a worldwide perspective. J Natl Cancer Inst 1995;87:796-802. http://www.ncbi.nlm. nih.gov/pubmed/7791229 (accessed 14 January 2010).

23. McKenzie ND, Kobetz EN, Hnatyszyn J, Twiggs LB, Lucci JA. Women with HIV are more commonly infected with non-16 and -18 high-risk HPV types. Gynecol Oncol 2010;116(3):572.

24. Paavonen J, Naud P, Salmerón J, et al. Efficacy of human papillomavirus (HPV)-16/18 AS04-adjuvanted vaccine against cervical infection and precancer caused by oncogenic HPV types (PATRICIA): final analysis of a double-blind, randomised study in young women. Lancet 2009:374:301-314. http://www.thelancet.com/journals/lancet/article/PIIS01406736(09)61248-4/fulltext (accessed 20 November 2009).

25. Schelcht NF, Bick RD, Palefsky JM, et al. Variants of human papillomavirus 16 and 18 and their natural history in human immunodeficiency virus-positive women. J Gen Virol 2005:86:2709-2720. http://vir.sgmjournals.org/cgi/reprint/86/10/2709.pdf (accessed 20 November 2009)

26. Qiao Y-L, Sellors JW, Eder PS, et al. A new HPV-DNA test for cervical-cancer screening in developing regions: a cross-sectional study of clinical accuracy in rural China. Lancet Oncol 2008;9(10):929-936. http://www.thelancet.com/journals/lanonc/article/ PIIS1470204508702109/abstrac\#\# (accessed 15 January 2010).

27. Mwanahamuntu MH, Sahasrabuddhe VV. Implementation of 'see-and-treat' cervical cancer prevention services linked to HIV care in Zambia. AIDS 2009;23(6):N1-N5. http://www. medscape.com/viewarticle/705167 (accessed 1 July 2010). 